\title{
Mapping femtosecond pulse front distortion and group velocity dispersion in multiphoton microscopy
}

\author{
I. D. C. Tullis, S. M. Ameer-Beg, P. R. Barber, V. Rankov and B. Vojnovic \\ Advanced Technology Development Group, Gray Cancer Institute, Mount Vernon Hospital, \\ Northwood, United Kingdom, HA6 2JR
}

\begin{abstract}
Group velocity dispersion (GVD) and pulse front distortion of ultrashort pulses are of critical importance in efficient multiphoton excitation microscopy. Since measurement of the pulse front distortion due to a lens is not trivial we have developed an imaging interferometric cross-correlator which allows us to measure temporal delays and pulse-widths across the spatial profile of the beam. The instrument consists of a modified Michelson interferometer with a reference arm containing a voice-coil delay stage and an arm which contains the optics under test. The pulse replicas are recombined and incident on a $22 \times 22$ lenslet array. The beamlets are focused in a $0.5 \mathrm{~mm}$ thick BBO crystal (cut for Type I second harmonic generation), filtered to remove the IR component of the beam and imaged using a $500 \mathrm{fps}$ camera. The GVD and pulse front distortion are extracted from the temporal stack of beamlet images to produce a low resolution spatio-temporal map.
\end{abstract}

Keywords: Group velocity dispersion, multiphoton microscopy

\section{INTRODUCTION}

Visualization and measurement of dynamic processes within living cells and tissues without significant perturbation of the specimen is a significant problem in biological imaging. Both widefield epi-fluorescence and confocal microscopies can provide adequate spatial resolution and are commonplace and easy to use, however; these techniques are limited in sensitivity due to scattering of the excitation illumination and by extensive photobleaching of the fluorophore.

Multiphoton microscopy offers significant advantages in this regard due to the non-linear nature of the excitation process. The excitation of fluorphores is limited to a small volume and results in a sectioning effect and reduced out of focus photobleaching; the longer excitation wavelengths allow deeper penetration into scattering samples.

The particular advantage of multiphoton imaging lies in the generation of images from deep within biological tissue. Imaging under such circumstances with conventional objectives is beyond their design specifications: a reduction in resolution is inevitable. ${ }^{1}$ An increase in the point spread function (PSF) and pulse broadening due to the optical train and sample itself leads to a reduction in delivered NIR intensity and therefore fluorescence emission. It is clear that the temporal and spatial deposition of the pulsed power needs to be maintained at depth through highly aberrant biological media in order to maintain both the optical PSF and detectable signal. Recently there have been a limited number of applications of adaptive optics (AO) in multiphoton microscopy ${ }^{2,3,4}$ and determinations of pulse broadening. ${ }^{5,6,7,8}$ The intertwined nature of these aspects have led us to consider the application of both $\mathrm{AO}$ and adaptive pulse compression (APC) approaches to multiphoton imaging in aberrant media. The implementation of adaptive pulse-compression system to pre-compensate for dispersion and optical aberration in the focal plane will enable both a-priori determined errors to be corrected and, by iterative correction, distortion due to microscopic and macroscopic features in the samples of interest. Intravital imaging of the tumour micro-environment with multiphoton microscopy enables determination of clinically relevant data including drug delivery, pharmaco-kinetics and, for example, long term mapping of angiogenesis (new blood vessel formation). Improvement in the penetration depth (currently $\approx 200 \mu \mathrm{m}$ for tumour) for imaging in millimeter size

Further author information: (Send correspondence to I.D.T.)

I.D.T.: E-mail: tullis@gci.ac.uk, Telephone: +44 1923828611 
tumour models in situ in order to avoid surface effects, whilst maintaining resolution is clearly required. Pulse front distortion mapping and correction in deep tissue imaging of tumour vascular networks/tumour metastasis will enable key physiological processes to be evaluated in situ with improved imaging resolution and depth.

The spatio-temporal profile of a femtosecond optical pulse is strongly affected by the dispersion properties of the optical element. The dispersion properties of the optical elements are well known ${ }^{9,10}$ and a variety of instruments have been developed to measure the spatial-temporal properties of an ultrafast pulse.

Femtosecond pulses propagating through a lens experience two kinds of distortions: the pulse front, which is defined as the surface coinciding with the peak of the pulse, moves with the group velocity and is thus delayed with respect to the phase front. The relative delay to the pulse front is dependent on the thickness of glass traversed and varies as a function of the input beam radius $r$. This effect will be referred to as propagation time difference $(\mathrm{PTD})^{9}$ and is proportional to the change in refractive index with wavelength $d n / d \lambda$. Pulses passing through the edge of a lens arrive at the focal plane earlier than those propagating through the center of the lens. ${ }^{11}$ The second distortion is a broadening due to group velocity dispersion (GVD) of the material of the lens. This effect is proportional to $d^{2} n / d \lambda^{2}$ of the material and is also dependent on the path length in the glass, therefore the GVD also varies as a function of $r$. Pulses passing through the edge of a lens arrive at the focal plane with less dispersion and are narrower than those propagating through the center of the lens. Spatial beam profiling, wavefront sensing and temporal autocorrelation are diagnostic techniques are used to quantify beam properties but these are usually applied sequentially. ${ }^{8}$

A number of techniques have been applied in an attempt to measure PTD in optical systems with a variety of success. If the optical system is known an analytical solution for PTD and GVD may be computed using the paraxial approximation. ${ }^{9}$ Guild et al measured the GVD of a variety Zeiss microscope objectives. ${ }^{8}$ In their experiments one pass of the objective is made and the reported GVD of a NeoFluar $\times 400.9 \mathrm{NA}$ objective is 1800 $\mathrm{fs}^{2}$ and Apochromat $\times 631.4 \mathrm{NA}$ is $3200 \mathrm{fs}^{2}$. Wolleschensky et al ${ }^{12}$ measure the on and off axis GVD of a variety Zeiss objectives and find a strong agreement of the results with theory.

Fittinghoff et al measured the bulk GVD of microscope objectives with a second harmonic generation (SHG) frequency-resolved optical gating (FROG) and were able to plot the group delay of the pulse against the normalized pupil coordinate using the cross-correlation between the central ray and outer rays. ${ }^{13}$ Netz et al ${ }^{14}$ and Ameer-Beg et al $^{15}$ describe a variety of Michaelson-autocorrelation methods to measure the GVD and wavefront of a ultrafast pulse. These methods enable sequential mapping of the pulse front distortion and could be applied to arbitrary distortions in an imaging mode.

Szatmári and Kühnle ${ }^{16}$ describe a shearing interferometer autocorrelator which can measure the spatial variation of GVD but interpretation of the interferogram may not be intuitive in certain instances. The most promising technique has been proposed by Grunwald et al ${ }^{17}$ who describe measuring the first and second order spatiotemporal autocorrelation maps of sub-10-fs localized wave packets using an array of refractive and reflective axicons.

To date most methods of pulse front distortion measurement have been unwieldy in application or interpretation and are slow to provide information. We propose in this paper a method based on a collinear interferometric cross-correlation technique for determining the spatial distortion of optical pulses from ultrashort laser oscillators. This technique has been used to map temporal distortions due to propagation in microscope objectives.

\section{EXPERIMENTAL TECHNIQUE}

Experiments were performed with femtosecond pulses from a mode locked Ti:Sapphire laser with a center wavelength of $790 \mathrm{~nm}$ and a repetition rate of $76 \mathrm{MHz}$. A Faraday isolator was used to reject any light backscattered from the experiment from entering the laser. The beam was expanded with two sequential telescopes to approximately $8 \mathrm{~mm}$ in diameter $\left(1 / e^{2}\right)$ to overfill the back aperture of the objective. Dispersion in the telescopes and the Faraday isolator broaden the input pulse to $300 \mathrm{fs}$.

A Michelson interferometer was built using a ultrafast 50:50 type broadband beamsplitter. The polarization of the input beam was controlled with a $\lambda / 2$ waveplate to equalize optical power in each arm of the interferometer. In the reference arm an electromagnetic translator moves a mirror in steps of $15 \mathrm{~nm}$ (corresponding to a lag of 0.10 
fs) synchronously with the frame clock of the camera. The advantage delay line autocorrelation measurements is that arbitrary precision can be achieved as long as the motion of the delay line is linear with time - delays less than $0.1 \mathrm{fs}$ can be introduced.

The sample arm contains two identical microscope objectives under test, aligned coaxially, in order to maximize the group velocity dispersion and pulse front distortion. Light makes four passes through the objective under test. A schematic of the experimental rig is shown in Figure 1 and a photograph of the experiment in Figure 2. The objectives tested are designed to be used in air, with coverslips, or immersed in water and the appropriate media was introduced at the at the focal plane (coverslips are shown in Figure 1).

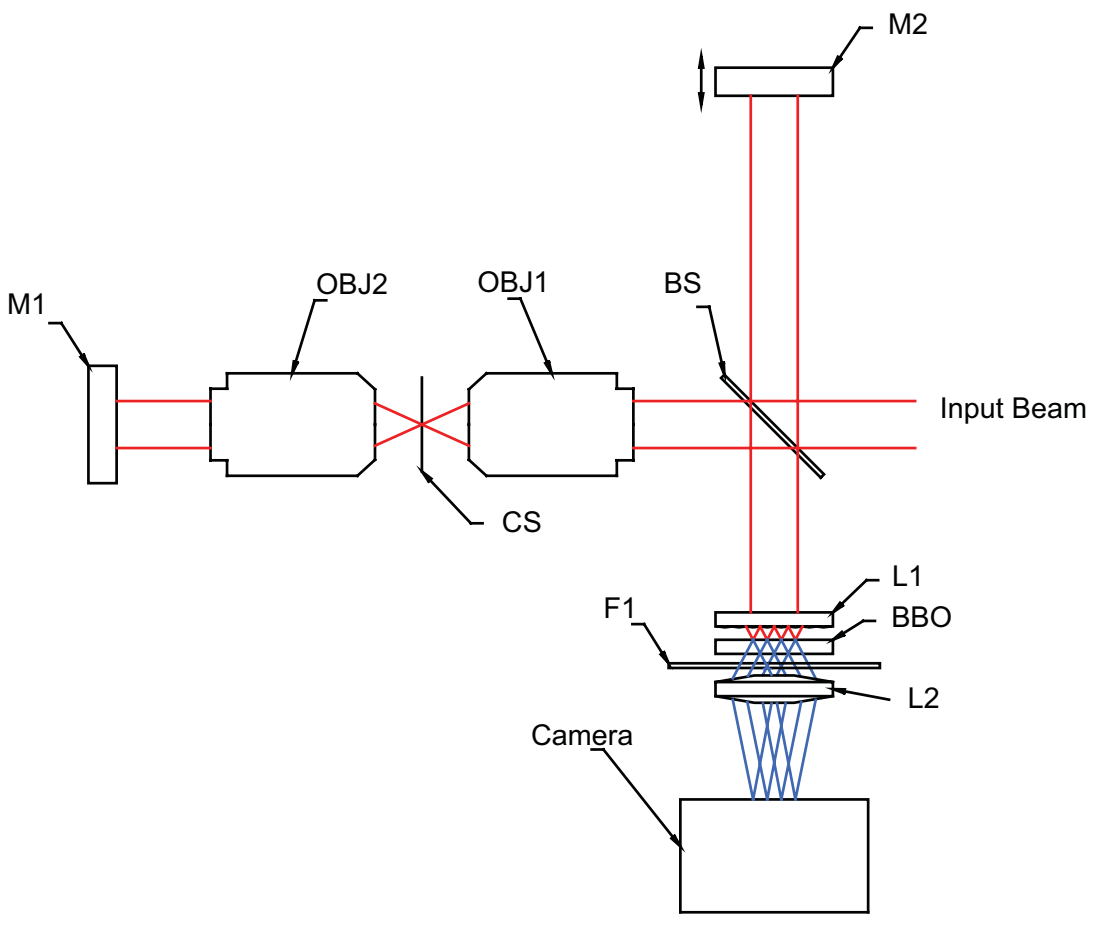

Figure 1. Schematic diagram of the autocorrelator. Light enters the system from the right from the Ti:Sapphire laser and is split into two paths by the beamsplitter (BS) configured as a Michelson interferometer. Light propagates through the test objective (OBJ1), a pair of coverslips (CS), a collimating objective (OBJ2) and is reflected back upon itself by mirror M1. A fraction of the beam travels up the reference arm and is reflected by moveable mirror M2 driven by a electromagnetic translator. A lenslet array (L1) focuses an array of recombined beams onto a BBO crystal. A filter (F1) removes any near infrared light and the lens L2 forms an image of the second harmonic signal on the fast frame rate camera.

The time delay between the wavepackets travelling in the reference arm and the sample arm could be coarsely controlled with a mirror mounted on a manual micrometer translation stage in the reference arm to enable the matching of the time delay in both arms and to allow the calibration of the electromagnet translator. The reference and the test wavefronts are recombined and the resulting beam is incident on a $22 \times 22$ array of hexagonally packed lenslets with a focal length of $6.70 \mathrm{~mm}$.

The beamlets are focused in a $0.5 \mathrm{~mm}$ thick BBO crystal (cut for Type I second harmonic generation) and filtered to remove the IR component of the beam and imaged onto the camera. The camera is based on the KAC-9630 CMOS image sensor which is arranged as an array of $128 \times 98$ pixels with a $20 \mu \mathrm{m}$ pitch. The integration time was set to $\approx 300 \mu$ s and read out at a frame rate of $500 \mathrm{~Hz}$. The sensor has an on board video gain amplifier, offset control and an 8-bit on-chip ADC. For each experiment the gain and offset were adjusted to give a maximum signal output with a dark level of around 10 counts.

The electromagnetic translator was developed in house and based on a loudspeaker operated under computer control. The output of a 14 bit digital to analog converter was amplified in a push pull amplifier stage to drive 


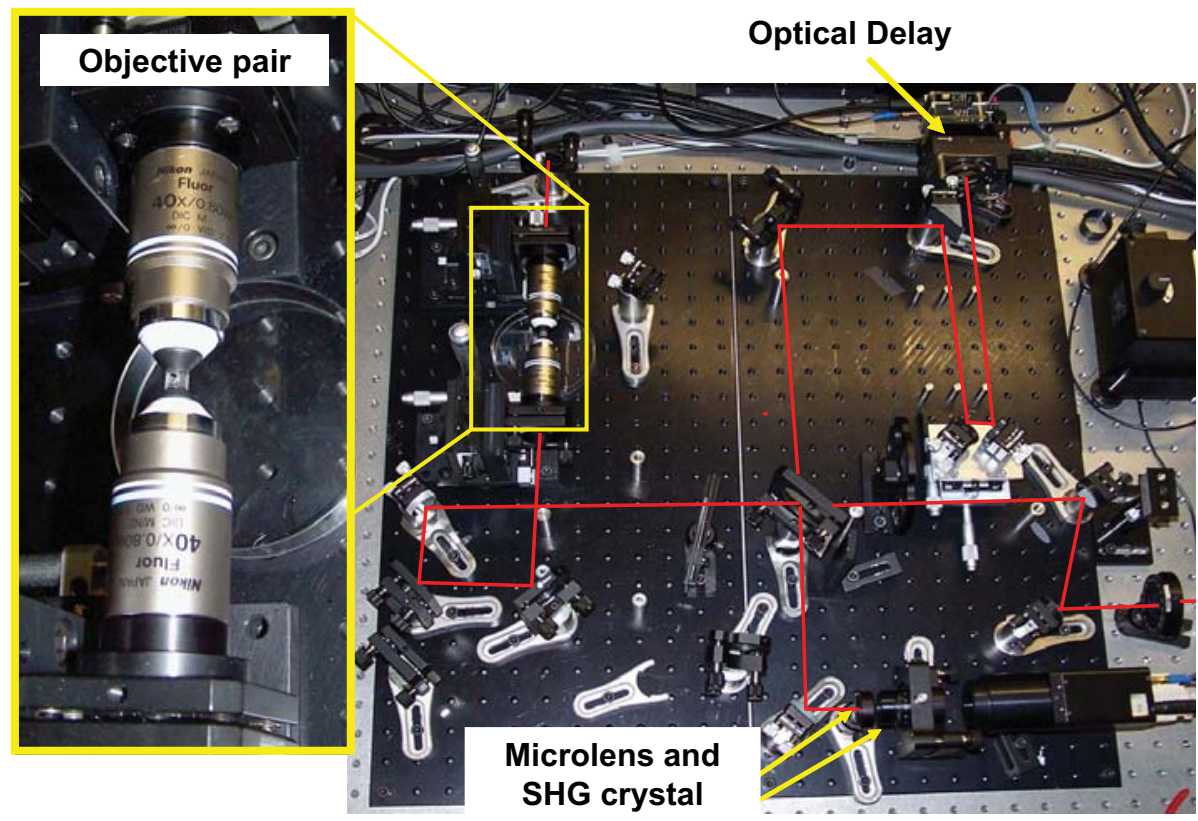

Figure 2. Photograph of the experimental layout. The objectives under test are arranged such that the probe beam is aberrated by four passes through the optics.

the loudspeaker coil. Position feedback, using a sliding vane optically interrupting the beam from a LED to a photodetector, was used to electronically stabilize the mirror position. A synchronizing pulse from the CMOS camera triggered the microcontroller to advance the mirror position.

The objectives were aligned so that the recombined beam produced the largest bullseye interference pattern on the camera possible. Under software control, 16384 images were acquired from the camera to record the interferogram with lags from -820 fs to $+820 \mathrm{fs}$.

The data were filtered in the time dimension to reduce the interferometric autocorrelation to its envelope and a least squares fit made to theory for a sech ${ }^{2}$ pulse. ${ }^{18}$ From the fitting parameters, the pulse width (FWHM), $\Delta t_{o}$, and the temporal position of the pulse peak may be derived.

A sample of the beam in the reference arm was picked off and input into a commercial autocorrelator in order to determine the input pulse width, $\Delta t$. The $\mathrm{GVD}^{2}$ in each objective was computed from the output pulse width $\Delta t_{o}$ using the following equation:

$$
\Delta t_{o}=\sqrt{\Delta t^{2}+4\left(\mathrm{GVD}^{2}\right)}
$$

A convex hull of the peak position and the GVD ${ }^{2}$ was plotted.

\section{RESULTS}

The temporal autocorrelation from one microlens of the array is plotted in Figure 3 and shows a typical 8:1 peak-to-background ratio.

Figure 4 shows a test experiment of pulse front delay without any objectives in the sample arm. The reference plot was produced by aligning the bullseye of the interference pattern with the camera. There is a slight edge effect, in the lower right hand side of the image, caused by the exclusion of data from some pixels that did not meet the appropriate signal-to-noise criteria. When the wavefront is tilted, the plot clearly shows the increasing pulse front delay from right to left. Figure 5 shows the plot of pulse front distortion and and the one pass GVD for one of the objectives tested, a water immersion Nikon Fluor $40 \times 0.8$ NA. 
The high spatial frequency variation of pulse arrival time and GVD across the pupil of the objective was unexpected. This may be due to mis-alignment of the objectives in our experimental setup or may be simply due to contaminants on the optical surfaces as the objectives have been used in a busy laboratory for several years. The measured value of GVD compares well with the expected values.

A variety of commonly used microscope objectives as listed in Table 1 was examined.

\begin{tabular}{llllll}
\hline Objective & Magnification & NA & $\begin{array}{l}\text { Working } \\
\text { Distance }(\mathrm{mm})\end{array}$ & GVD $\left(\mathrm{fs}^{2}\right)$ & Notes \\
\hline Nikon Plan Fluor & $10 \times$ & 0.3 & 16 & 20,000 & \\
Nikon Fluor & $40 \times$ & 0.8 & 4 & 8000 & Water dipping \\
Nikon Plan Fluor & $40 \times$ & 0.6 & 2.9 & 8000 & ELWD Coverslip correction \\
\hline
\end{tabular}

Table 1. Microscope objectives tested.

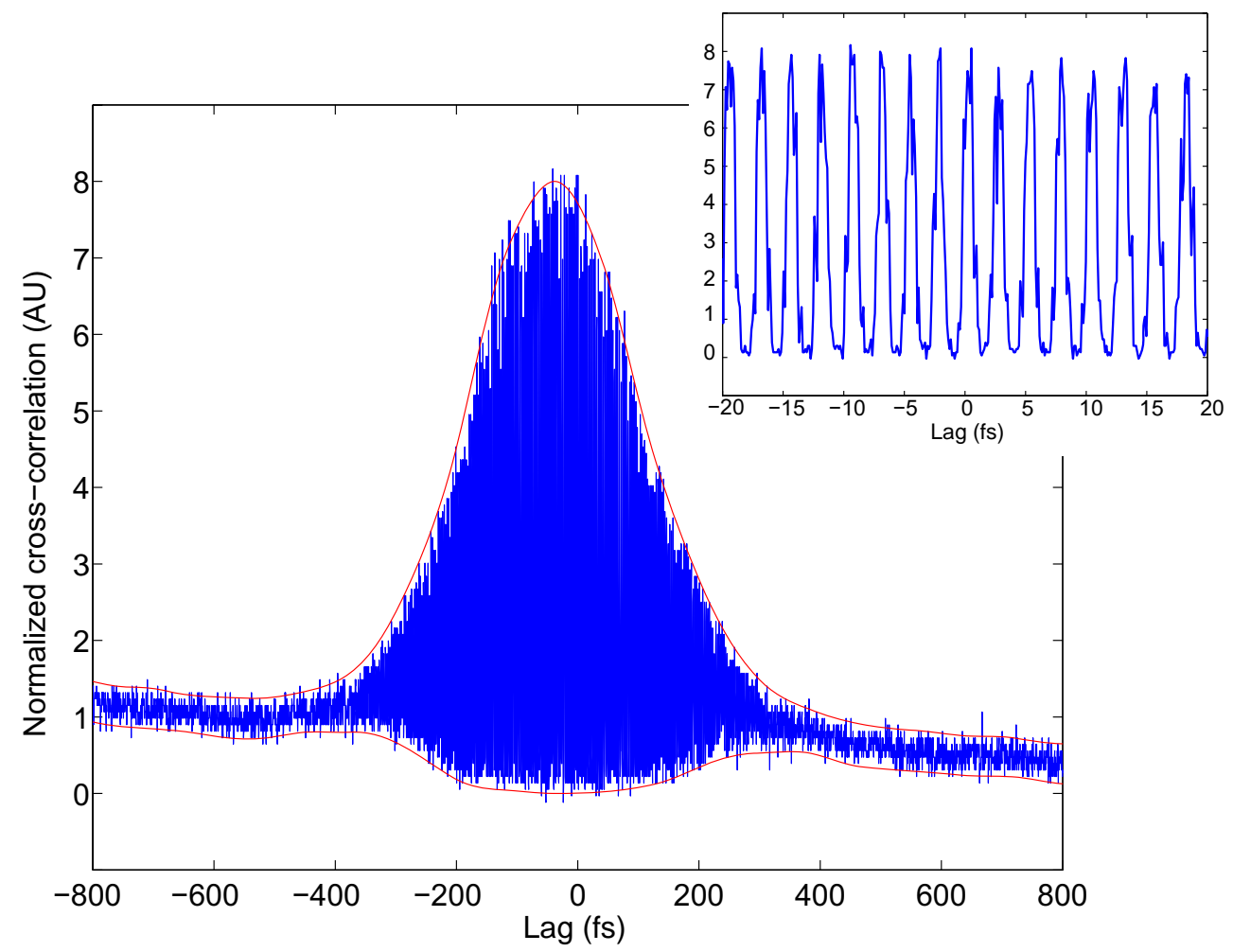

Figure 3. Plot of time course from one pixel of camera output. The insert shows an expanded view of cross-correlation from the central region. The intensity has been normalized with respect to the baseline second harmonic generation, to show the 8:1 peak-to-background ratio.

\subsection{Future Work}

The pulse peak position and the GVD could be estimated with greater resolution if the optical pulses entering the autocorrelator are of shorter width. A prism pair pulse compressor is currently being constructed to prechirp the optical pulses exiting the laser which should result in a shorter pulse at the autocorrelator. We are also currently evaluating more microscope objectives and plan to measure the pulse front distortion and GVD 


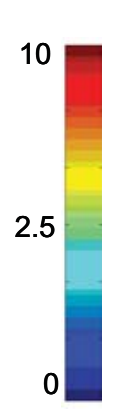

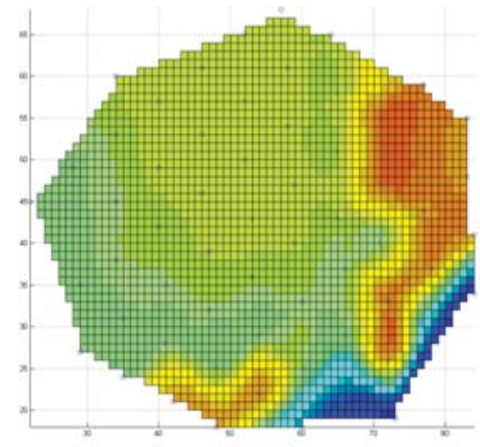

Reference

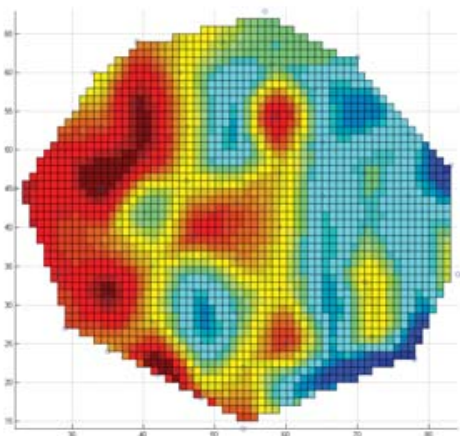

Applied Tilt/Tip

\section{Pulse Front Delay (fs)}

Figure 4. A map of pulse front distortion with no objective in place (left) and after a static tilt/tip is applied (right).

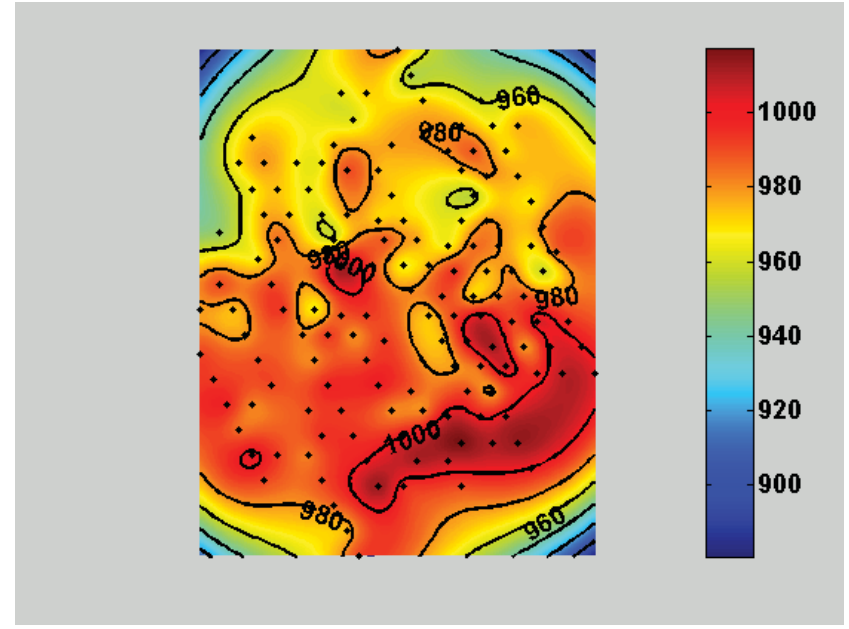

Pulse front delay (fs)

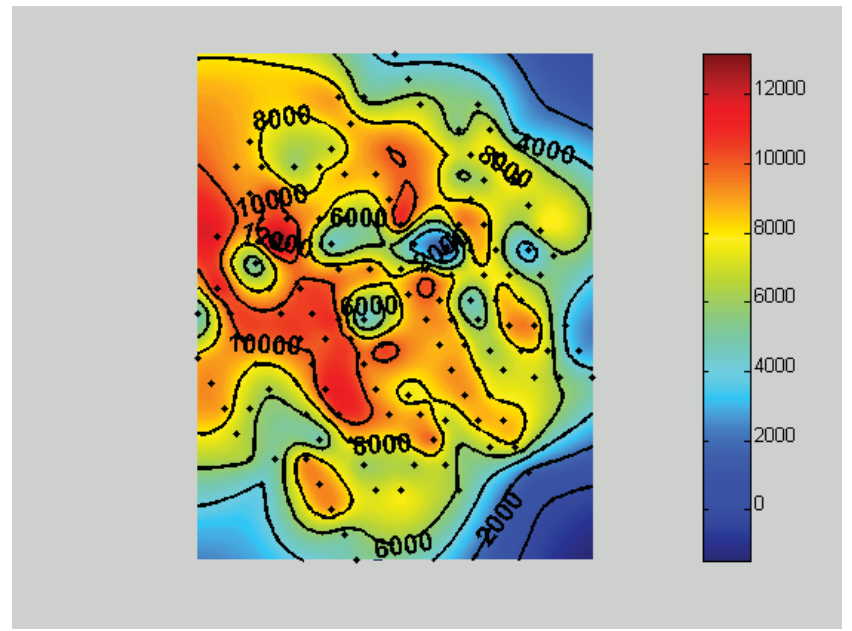

One pass $\mathrm{GVD}^{2} \quad\left(\mathrm{fs}^{2}\right)$

Figure 5. Pulse front distortion and $\mathrm{GVD}^{2}$ for a Nikon $40 \times$ objective.

of samples placed in the focal plane between the objectives. Information from maps (Figure 5) will be used to correct for abberation thus introduced.

\section{CONCLUSIONS}

The measurement of the wavefront temporal delay and pulse width across the aperture of a microscope objective has been demonstrated. Combining the position of the focal spot in the camera image, with appropriate calibration images, would allow the measurement of the wavefront with a Shack-Hartmann-like technique. Additional information is available in the interferometric autocorrelation which is currently discarded in the data processing. Control of the spatial-temporal profile of an ultrashort pulse would find applications in coherent control, micromachining and optical microscopy. ${ }^{17}$ 


\section{ACKNOWLEDGMENTS}

This research was supported by "Deep, time-resolved imaging in aberrant biological media by adaptive and predictive wave-front correction," UK Research Councils Basic Technology Program (GR-R87901-01) and by Cancer Research UK Programme Grant C133-A1812

\section{REFERENCES}

1. M. J. Booth and T. Wilson, "Refractive-index-mismatch induced aberrations in single-photon and twophoton microscopy and the use of aberration correction," Journal of Biomedical Optics 6(3), pp. 266-272, 2001.

2. L. Sherman, J. Y. Ye, O. Albert, and T. B. Norris, "Adaptive correction of depth-induced aberrations in multiphoton scanning microscopy using a deformable mirror," Journal of Microscopy 206(1), pp. 65-71, 2002.

3. M. A. A. Neil, R. Juskaitis, M. J. Booth, T. Wilson, T. Tanaka, and S. Kawata, "Adaptive aberration correction in a two-photon microscope," Journal of Microscopy 200(2), pp. 105-108, 2000.

4. A. J. Wright, B. A. Patterson, S. P. Poland, J. M. Girkin, G. M. Gibson, and M. J. Padgett, "Dynamic closed-loop system for focus tracking using a spatial light modulator and a deformable membrane mirror," Optics Express 14(1), pp. 222-228, 2006.

5. M. Müller, J. Squier, and G. J. Brakenhoff, "Measurement of femtosecond pulses in the focal point of a high-numerical-aperture lens by two-photon absorption," Optics Letters 20(9), p. 1038, 1995.

6. M. Müller and G. J. Brakenhoff, "Characterization of high-numerical-aperture lenses by spatial autocorrelation of the focal field," Optics Letters 20(21), p. 2159, 1995.

7. C. Radzewicz, M. l. Grone, and J. Krasinski, "Interferometric measurement of femtosecond pulse distortion by lenses," Optics Communications 126, pp. 185-190, 1996.

8. J. B. Guild, C. Xu, and W. W. Webb, "Measurement of group delay dispersion of high numerical aperture objective lenses using two-photon excited fluorescence," Applied Optics 36(1), pp. 397-401, 1997.

9. Z. Bor, "Distortion of femtosecond laser pulses in lenses and lens systems," Journal of Modern Optics 35(12), pp. 1907-1918, 1988.

10. Z. Bor, "Distortion of femtosecond laser pulses in lenses," Optics Letters 14(2), pp. 119-121, 1989.

11. M. Kempe and W. Rudolph, "Impact of chromatic and spherical aberration on the focusing of ultrashort light pulses by lenses," Optics Letters 18(2), p. 137, 1993.

12. R. Wolleschensky, T. Feurer, R. Sauerbrey, and U. Simon, "Characterization and optimization of a laserscanning microscope in the femtosecond regime," Applied Physics B 67, pp. 87-94, 1998.

13. D. N. Fittinghoff, A. C. Millard, J. A. Squier, and M. Müller, "Frequency-resolved optical gating measurement of ultrashort pulses passing through a high numerical aperture objective," IEEE Journal of Quantum Electronics 35(4), pp. 479-486.

14. R. Netz, T. Feurer, R.Wolleschensky, and R. Sauerbrey, "Measurement of the pulse-front distortion in high-numerical-aperture optics," Applied Physics B 70, pp. 833-837, 2000.

15. S. Ameer-Beg, A. Langley, I. Ross, W. Shaikh, and P. Taday, "An achromatic lens for focusing femtosecond pulses: direct measurement of femtosecond pulse front distortion using a second-order autocorrelation technique," Optics Communications 122(4), pp. 99-104, 1996.

16. S. Szatmári and G. Kühnle, "Pulse front and pulse duration distortion in refractive optics, and its compensation," Optics Communications 69(1), pp. 60-65, 1988.

17. R. Grunwald, U. Neumann, U. Griebner, K. Reimann, G. Steinmeyer, and V. Kebbel, "Ultrashort-pulse wave-front autocorrelation," Optics Letters 28(23), pp. 2399-2401, 2003.

18. J.-C. M. Diels, J. J. Fontaine, I. C. McMichael, and F. Simoni, "Control and measurement of ultrashort pulse shapes (in amplitude and phase) with femtosecond accuracy," Applied Optics 24(9), pp. 1270-1282, 1985. 